\title{
Dynamical changes in hydration water accompanying lysozyme thermal denaturation
}

\author{
Francesco Mallamace ${ }^{1,2,3, \dagger}$, Carmelo Corsaro ${ }^{1,2}$, Domenico Mallamace ${ }^{4}$, Nicola Cicero ${ }^{4}$, \\ Sebastiano Vasi ${ }^{2}$, Giacomo Dugo ${ }^{4}$, H. Eugene Stanley ${ }^{3}$ \\ ${ }^{1}$ CNR-IPCF Messina, Istituto per i Processi Chimico-Fisici, Viale F. Stagno D'Alcontres 37, 98158 Messina, Italy \\ ${ }^{2}$ Dipartimento di Fisica e Scienze della Terra, Università di Messina, Viale F. Stagno D'Alcontres 31, 98166 Messina, Italy \\ ${ }^{3}$ Center for Polymer Studies and Department of Physics, Boston University, Boston, MA 02215, USA \\ ${ }^{4}$ Dipartimento di Scienze dell'Ambiente, della Sicurezza, del Territorio, degli Alimenti, e della Salute, \\ Università di Messina Viale F. Stagno d'Alcontres 31, 98166 Messina, Italy \\ Corresponding author. E-mail: †francesco.mallamace@unime.it \\ Received July 7, 2015; accepted August 15, 2015
}

\begin{abstract}
We study the dynamics of the first hydration shell of lysozyme to determine the role of hydration water that accompanies lysozyme thermal denaturation. We use nuclear magnetic resonance spectroscopy to investigate both the translational and rotational contributions. Data on proton self-diffusion and reorentational correlation time indicate that the kinetics of the lysozyme folding/unfolding process is controlled by the dynamics of the water molecules in the first hydration shell. When the hydration water dynamics change, because of the weakening of the hydrogen bond network, the three-dimensional structure of the lysozyme is lost and denaturation is triggered. Our data indicates that at temperatures above approximately $315 \mathrm{~K}$, water behaves as a simple liquid and is no longer a good solvent.
\end{abstract}

Keywords lysozyme unfolding, hydration water, NMR, correlation time, solvent dynamics

PACS numbers 87.15.Kr, 76.60.Es, 87.15.hg

\section{Introduction}

Proteins in their unfolded state are a linear chain of amino acids without a stable three-dimensional structure $[1,2]$. Under the right thermodynamic conditions, such as temperature and hydration, amino acids follow a precise physical route and rapidly evolve into a characteristic protein structure - a native protein state [3]. The bio-functionality of a protein is directly linked to the amino acid sequence and structure that characterizes the native protein state; furthermore, it is strongly dependent on the solvent, i.e., water, which actually functions as an amino acid [4]. Dry proteins exhibit no biological activity and require at least a water monolayer (a first hydration shell) covering their surface to function as proteins [5-7]. Water in the form of internal water is also an essential part of the three-dimensional structure of a protein $[8,9]$.

Many studies have probed the thermal limit of the biological activity of protein and have linked its onset at low temperatures to the occurrence of a dynamical crossover in the hydration water at approximately $225 \mathrm{~K}$ [10-20]. Although this precise value of temperature has been debated, given the possibility of inadequate instrumental resolutions, there is a consensus that the crossover occurs and that it is important [21-31].

The hydrophobic interaction is an important driving force in the aggregation and folding processes in proteins [32], in particular, in the burial effect of amino acid residues in protein interiors [33]. However, the process that maintains their stability and functionality is the hydrophilic interaction $[27,34,35]$. The hydrogen bonds (HB) formed between hydrophilic protein groups and water molecules are vital to protein life [4]. In addition, the thermodynamic properties of water, governed by the lifetime and stability of the HBs, influence the protein activity $[27,28,36]$ to the extent that the slaving concept and model is used to describe how solvent fluctuations determine protein dynamics and functions $[37,38]$. For example, the temperature above which water is no longer a good solvent and loses its anomalous

*Special Topic: Water Science (Eds. Li-Mei Xu \& En-Ge Wang).

(C) The Author(s) 2015. This article is published with open access at www.springer.com/11467 
fundamental properties is approximately $315 \mathrm{~K}$. Infact, the lifetime and stability of the HBs dramatically decrease with increasing temperature and above $T^{*} \simeq 315$ $\mathrm{K}$ water behaves as a simple liquid [39].

The folding/unfolding process of proteins is controlled by variables such as $\mathrm{pH}$, temperature, solvent, and time $[6,40,41]$. Recent studies on the hydrated lysozyme have determined the characteristic temperatures of the process. Although the protein begins to lose its threedimensional structure above $315 \mathrm{~K}$, if the temperature of the system can be maintained below $T_{D}=346 \mathrm{~K}$ for a short period of time, the unfolding mechanism can be stopped and reversed [42, 43].

The thermal denaturation of the lysozyme has been studied using such techniques as calorimetry, nuclear magnetic resonance (NMR), and Fourier transform infrared spectroscopy $[13,14,43,44]$. The process is kinetic and moves through three states: the native state $\leftrightarrow$ the reversible unfolding state $\rightarrow$ the irreversible denatured state. The first is initiated at $\sim 315 \mathrm{~K}$ and can be reversed under certain conditions and the second is initiated at $\sim 346 \mathrm{~K}$ and cannot be reversed.

The dynamics of the first hydration shell of water surrounding the protein surface is slower than the dynamics of the bulk solvent [45-47]. This is due to the complex network of hydrogen bonds that are formed between the water molecules and protein residues $[6,48,49]$. By increasing the temperature from the stable condition towards denaturation, however, the dynamics of hydration water change dramatically and trigger the unfolding process.

We use nuclear magnetic resonance to study the translational and rotational contributions made by the dynamics of lysozyme hydration water. The goal is to understand the dynamical changes that are responsible in driving protein denaturation.

In particular, we measure the translational contribution to the dynamics by studying the self-diffusion coefficient measured for hydrated lysozyme with a single hydration shell. In addition, we measure the spin-lattice relaxation time and extract the reorientational correlation time. We compare these parameters, evaluated for the hydration water, with the corresponding parameters for bulk water and find a strict connection between the dynamic water properties and the activity of a protein moving toward denaturation.

\section{Experiments}

To measure how the dynamics of the first hydration shell of water molecules surrounding the protein surface change, and to link it with the protein thermal limits of functioning (the unfolding process), we examined a protein lysozyme (a small protein of $14.4 \mathrm{kDa}$ ) with a hydration level (i.e., grams of water per gram of dry protein) of $h=0.3$. We purchased lysozyme samples from Fluka (L7651 three times crystallized, dialyzed, and lyophilized), dried them, and then hydrated them isopiestically [13].

We used NMR (a 700-MHz Bruker Avance spectrometer) at one atmosphere to measure the proton selfdiffusion coefficient of lysozyme hydration water [14, 28]. We performed the measurements using the pulsed field gradient stimulated echo (PGSTE) technique [50] with a field gradient value of 1200 Gauss/cm [14].

We also measured the proton spin-lattice relaxation time, $T_{1}$, focusing on the thermal region of the denaturation, $280 \mathrm{~K}<T<360 \mathrm{~K}[14,29]$. We used the inversion-recovery pulse sequence [51] to obtain the $T_{1}$ data and varied the interpulse delay from microseconds to several seconds. The data fit a weighted double exponential form,

$$
\frac{M}{M_{0}}=1-2\left[P \exp \left(-\frac{t}{T_{1 s}}\right)+(1-P) \exp \left(-\frac{t}{T_{1 f}}\right)\right],
$$

where $P$ is the weight, $t$ the interpulse delay variable, and $T_{1 s}$ and $T_{1 f}$ are the two relaxing contributions. Figure 1 shows the curve fitting for two different temperatures.

The slowest relaxing component $T_{1 s}$ (in the order of seconds) belongs to water protons in the hydration shell of the lysozyme, and the fastest relaxing component $T_{1 f}$ belongs to strongly-bonded protons, e.g., those of internal (or crystallization) water $[14,29]$. Note that the fastest contribution becomes progressively smaller at the highest temperatures and because the internal water exchanges with the hydration water when the protein unfolds, it has a weight of less than $10 \%$. Note that although

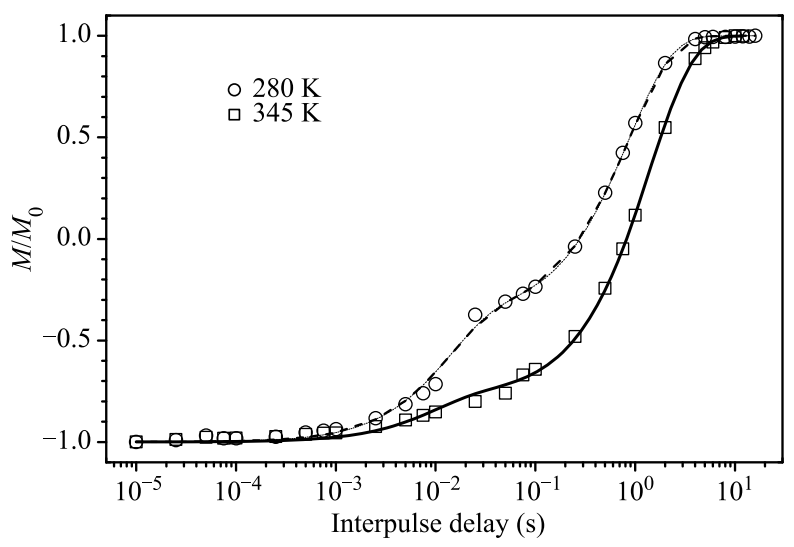

Fig. 1 Example of curve fitting after the execution of the inversion recovery pulse sequence for the determination of the spinlattice relaxation time. The fit was performed with Eq. (1). 
protein protons usually contribute to the NMR signal, under the experimental conditions in this study (and in particular low hydration), they are essentially immobile on the NMR observation time scale especially at low and ambient temperatures. Even with very high-resolution NMR techniques, at low hydration protein protons peaks are observable only for temperatures higher than the unfolding threshold and the magnitude of their intensity is about four orders smaller than that of the water peak [43]. In fact, in this case, in order to exclude the protein protons contribution, one can follow the relaxation corresponding just to the water peak.

\section{Results and discussion}

Although we know that the dynamics of water within the first hydration shell around the protein surface are slower than those of bulk water [45], the magnitude and molecular origin of this retardation are still topics for further study [46, 47, 52-55]. Fioretto et al. [52] used dielectric spectroscopy to measure the retardation coefficient, i.e., the ratio between the relaxation time of hydration water and the relaxation time of bulk water, and arrived at a value of 6-8 at ambient temperature [52].

Figure 2 shows in the bottom panel a log-linear plot of the ratio between the self-diffusion coefficient measured in bulk water $[56,57]$ and the self-diffusion coefficient measured in hydrated lysozyme with $h=0.3$ versus the inverse of the temperature. The original data are reported in the top panel of Fig. 2; they were interpolated in order to calculate the ratio at the same temperature.

Although the value we find at ambient temperatures

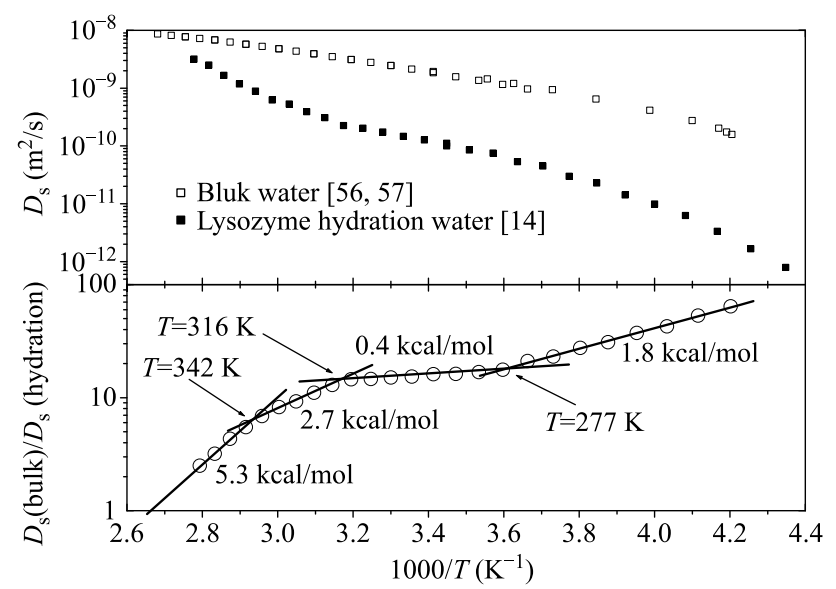

Fig. 2 The ratio between the self-diffusion coefficient measured in bulk water [56, 57] and that measured in hydrated lysozyme [43] as a function of the temperature (bottom panel), calculated from the interpolation of the original data reported in the top panel. Three variations are clearly visible in the obtained ratio. is higher than that found by Fioretto et al., a more interesting observation is that the Arrhenius plot (see the bottom panel of Fig. 2) indicates that three changes occur at approximately $277 \mathrm{~K}, 316 \mathrm{~K}$, and $342 \mathrm{~K}$. By comparing the individual behavior, reported in the top panel of Fig. 2, one can note that the observed kink in the ratio originates from changes in the temperature evolution of the hydration water.

As we increase the temperature, the first change occurs at $T=277 \mathrm{~K}$, which corresponds to the temperature at which bulk water has the maximum density. When $T<277 \mathrm{~K}$, the activation energy is $\approx 1.8 \mathrm{kcal} / \mathrm{mol}$, which is a typical hydrogen bond value. In this region the hydrogen bond network on the protein surface becomes progressively strong and stable. The region of optimal biological functioning between $277 \mathrm{~K}$ and $316 \mathrm{~K}$ (at which the second change occurs) shows a smaller activation en$\operatorname{ergy}(\sim 0.4 \mathrm{kcal} / \mathrm{mol})$. The $\approx 315 \mathrm{~K}$ temperature is the border temperature above which the hydrogen bond interaction weakens and water is no longer able to keep the protein in a folded state [39]. At temperature above $316 \mathrm{~K}$, water is no longer a good solvent and the hydrogen bonded network of the first hydration shell begins to crumble and triggers the protein unfolding process. In the region between $316 \mathrm{~K}$ and $342 \mathrm{~K}$, the activation energy is $\approx 2.7 \mathrm{kcal} / \mathrm{mol}$ and, depending on the duration, the unfolding process is reversible $[43,58]$. At the highest activation energy $(\sim 5.3 \mathrm{kcal} / \mathrm{mol})$, the unfolding process is irreversible. When $T>342 \mathrm{~K}$, the hydrogen bond network is disrupted and the structure of the protein resembles that of a linear chain of amino acids. Note that the ratio between the self-diffusion coefficients of bulk and hydration water tends toward 1 , which is the same as in bulk water for temperatures approaching the boiling point of bulk water. This implies that all water molecules are in a bulk condition and free to move.

Next, we examine the rotational dynamics by evaluating the reorientational correlation time using the measured self-diffusion and spin-lattice relaxation times. Assuming that the spin-rotation contribution to the proton relaxation rate is in the order of milliseconds at 360 $\mathrm{K}$ [59], and even shorter when below that temperature, only dipolar interactions can be taken into account for water relaxation and the observed proton relaxation can be written as the sum of inter- and intra-molecular contributions [60, 61],

$$
\left(\frac{1}{T_{1}}\right)_{\text {obs }}=\left(\frac{1}{T_{1}}\right)_{\text {inter }}+\left(\frac{1}{T_{1}}\right)_{\text {intra }} .
$$

The intermolecular term can be evaluated by using the self-diffusion coefficient $D_{s}[60]$, as 


$$
\begin{aligned}
\left(\frac{1}{T_{1}}\right)_{\text {inter }} & =\frac{N \pi \gamma^{4} \hbar^{2}}{5 a D_{s}}\left[1+0.233\left(\frac{b}{a}\right)^{2}\right. \\
& \left.+0.15\left(\frac{b}{a}\right)^{4}+\cdots\right]
\end{aligned}
$$

where $N$ is the number density of the nuclei, $\gamma$ the proton gyromagnetic ratio, $\hbar$ the Planck constant divided by $2 \pi, a$ the molecular hydrodynamic radius, and $b$ the distance of the proton from the center of the molecule.

At first approximation, for small time windows, the correlation function of protein hydration water can be considered exponential [62]. Therefore, in the extreme narrowing limit, i.e., when the product of the resonance frequency of the proton $\left(\omega_{0}\right)$ and the reorientational correlation time $\left(\tau_{\theta}\right)$ is much less than one $\left(\omega_{0} \tau_{\theta} \ll 1\right)$, it can be written as $[60,61]$,

$$
\left(\frac{1}{T_{1}}\right)_{\text {intra }}=\frac{3}{2} \frac{\gamma^{4} \hbar^{2}}{r^{6}} \tau_{\theta}
$$

where $r$ is the distance between the two protons in the water molecule. Thus, if we measure $D_{s}$ and $T_{1}$, we can evaluate the intra-molecular term and the reorientational correlation time $\tau_{\theta}$. Because we are focusing on the hydration water, we evaluate $\tau_{\theta}$ by considering the $T_{1 s}$ values and by assuming the constant values $a=1.38 \AA$, $b=0.92 \AA$, and $r=1.52 \AA[60]$.

Figure 3 compares the thermal behaviors of $\tau_{\theta}$ in lysozyme hydration water measured by NMR $(h=0.3)$, dielectric spectroscopy $(h=0.37)[24]$, and neutron scattering $(h=0.4)[25]$, with that of bulk water at ambient pressure [57] and emulsified water at 2 kbar [61]. Note that when the temperature drops below the temperature of irreversible denaturation, the reorientational correlation time in hydration water is slower than in bulk

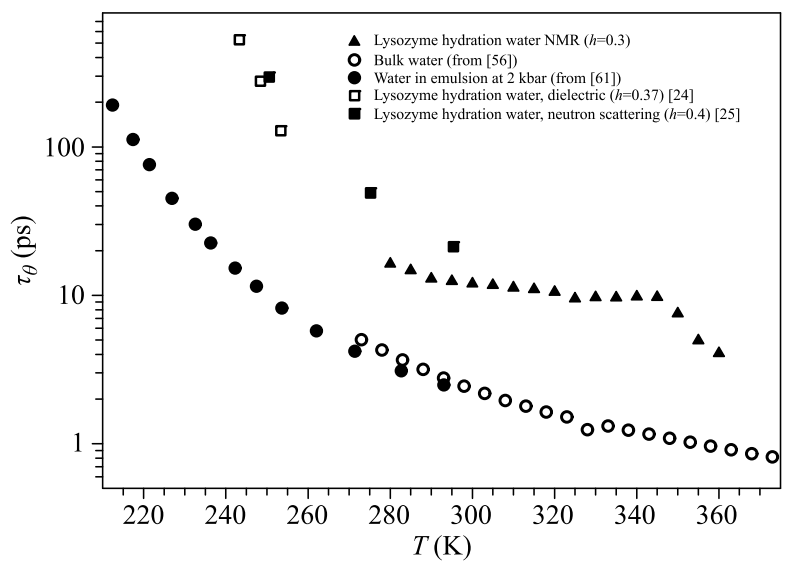

Fig. 3 The reorientational correlation time, $\tau_{\theta}$, of lysozyme hydration water measured by NMR $(h=0.3)$, dielectric spectroscopy $(h=0.37)$, and neutron scattering $(h=0.4)$, compared with that of bulk water at ambient pressure and to emulsified water at 2 kbar. water, but when $T>346 \mathrm{~K}$, the rotational dynamic values increase sharply and approach those of bulk water. This confirms the previous findings that when $T>346$ $\mathrm{K}$ the hydrogen bonded network breaks down and water molecules move freely.

Our analysis can be used to differentiate between two different dynamical degrees of freedom of hydration water. The intra-molecular dynamics is linked with the single molecule rotation about its own axis (self rotation), whereas the inter-molecular dynamics depends on the water interaction with protein molecules. Because the orientational correlation time is in the order of picoseconds, water molecules on the protein surface are highly mobile; however, their diffusion is slowed down by their interaction with protein amino acids (resulting in an obstruction factor). By applying the Stokes-Einstein relation [63] we can estimate the hydrodynamic radius of the diffusing particles,

$$
D_{s}=\frac{k_{\mathrm{B}} T}{6 \pi \eta \xi}
$$

where $k_{\mathrm{B}}$ is the Boltzmann constant, $\eta$ the shear viscosity, and $\xi$ the hydrodynamic radius (Fig. 4). It was shown that although the density of the first hydration shell of water around the protein surface is up to $20 \%$ higher than that of bulk water, the viscosity remains essentially the same [63]. Therefore, we evaluated the hydrodynamic radius by using the viscosity data of bulk water from the NIST database [64].

Note that when the temperature is low, the value of $\xi$ $(\sim 1.8 \mathrm{~nm})$ coincides with that of the hydrodynamic radius of lysozyme [54] confirming that the first hydration shell of water is hydrogen bonded with the hydrophilic groups of the protein surface. When $T>320 \mathrm{~K}$, however, $\xi$ rapidly decreases, the water molecules are no longer tightly bonded to the protein surface. They form local

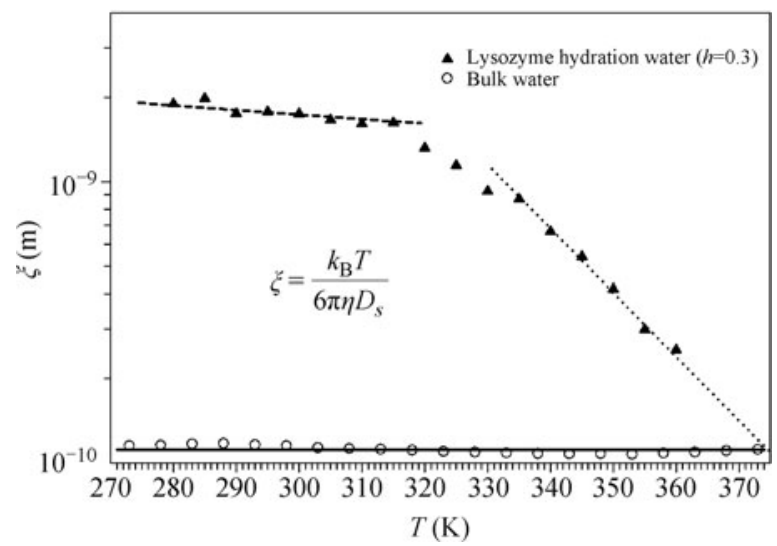

Fig. 4 The hydrodynamic radius obtained using the StokesEinstein relation for bulk water and lysozyme hydration water. Lines are guides for the eye. 
and transient aggregates whose dimension progressively decreases as the temperature increases. Confirming this, when $T \gtrsim 345 \mathrm{~K}$ and the protein unfolds completely, the $\xi$ values of hydration water approach those of bulk water (dotted line in Fig. 4).

\section{Conclusion}

We studied the dynamics of water in the first hydration shell of lysozyme and linked the corresponding changes to different states of the folding/unfolding process. We used nuclear magnetic resonance spectroscopy to differentiate between the translational and rotational contributions, which are the proton self-diffusion coefficient and the reorientational correlation time, respectively.

The self-diffusion in the hydration water is slower than in the bulk solvent, and the ratio between these two values (a retardation coefficient) as a function of the inverse temperature, shows three changes at approximately 277 $\mathrm{K}, 316 \mathrm{~K}$, and $342 \mathrm{~K}$, with corresponding changes in the activation energy (see Fig. 2). When $T<277 \mathrm{~K}$, the hydrogen bond network on the protein surface is strong and stable and the activation energy is $\approx 1.8 \mathrm{kcal} / \mathrm{mol}$. The region between $277 \mathrm{~K}$ and $316 \mathrm{~K}$ is the thermal region of optimal biological functioning and exhibits a smaller activation energy $(\sim 0.4 \mathrm{kcal} / \mathrm{mol})$. Above $\sim 315 \mathrm{~K}$, the hydrogen bonded network of the first hydration shell begins to crumble and the protein unfolding process begins. However, when the temperature is $316 \mathrm{~K}<T<342$ $\mathrm{K}$ and the activation energy is $\approx 2.7 \mathrm{kcal} / \mathrm{mol}$, the unfolding process is still reversible [43, 58]. Finally, when $T \gtrsim 342 \mathrm{~K}$ and the activation energy is $\sim 5.3 \mathrm{kcal} / \mathrm{mol}$, the unfolding process becomes irreversible, the hydrogen bond network breaks down completely, and the protein structure resembles that of a linear chain of amino acids.

In our rotational dynamics study, we found that for temperatures lower than the temperature of irreversible denaturation, the reorientational correlation time $\tau_{\theta}$ in hydration water is slower than in bulk water (see Fig. 3). When $T \sim 346 \mathrm{~K}, \tau_{\theta}$ sharply decreases and approaches the values for bulk water, and the water molecules can rotate freely.

Finally, we applied the Stokes-Einstein relation to the lysozyme hydration water and calculated the hydrodynamic radius of the particles (see Fig. 4). We found that at ambient temperatures the radius coincides with the lysozyme hydrodynamic radius $(\approx 1.8 \mathrm{~nm})$. When $T>320 \mathrm{~K}, \xi$ decreases sharply and when $T>345 \mathrm{~K}$ its value approaches that of bulk water. This result confirms that when lysozyme unfolds completely, the water molecules are essentially free and no longer bridge the protein residues.

Open Access This article is distributed under the terms of the Creative Commons Attribution License which permits any use, distribution, and reproduction in any medium, provided the original author(s) and the source are credited.

\section{References and notes}

1. G. R. Bowman and V. S. Pande, Protein folded states are kinetic hubs, Proc. Natl. Acad. Sci. USA 107, 10890 (2010)

2. G. D. Rose, P. J. Fleming, J. R. Banavar, and A. Maritan, A backbone-based theory of protein folding, Proc. Natl. Acad. Sci. USA 103(45), 16623 (2006)

3. M. Karplus, Behind the folding funnel diagram, Nat. Chem. Biol. 7, 401 (2011)

4. P. Ball, Water as an active constituent in cell biology, Chem. Rev. 108, 74 (2008)

5. J. A. Rupley, P. H. Yang, and G. Tollin, Thermodynamic and related studies of water interacting with proteins in $w a-$ ter in polymers, Vol. 127, edited by S. P. Rowland, ACS Symposium Series, 1980, p. 111

6. R. B. Gregory, Protein Solvent Interaction, New York: Marcel Dekker, 1995

7. L. Comez, S. Perticaroli, M. Paolantoni, P. Sassi, S. Corezzi, A. Morresi, and D. Fioretto, Concentration dependence of hydration water in a model peptide, Phys. Chem. Chem. Phys. 16, 12433 (2014)

8. J. A. Rupley and G. Careri, Protein hydration and function, Adv. Protein Chem. 41, 37 (1991)

9. V. Helms, Protein dynamics tightly connected to the dynamics of surrounding and internal water molecules, ChemPhysChem 8, 23 (2007)

10. G. Schirò, M. Fomina, and A. Cupane, Communication: Protein dynamical transition vs. liquid-liquid phase transition in protein hydration water, J. Chem. Phys. 139, 121102 (2013)

11. K. L. Ngai, S. Capaccioli, and N. Shinyashiki, The protein glass transition and the role of the solvent, J Phys Chem B 112(12), 3826 (2008)

12. K. L. Ngai, S. Capaccioli, and A. Paciaroni, Nature of the water specific relaxation in hydrated proteins and aqueous mixtures, Chem. Phys. 424, 37 (2013)

13. S.-H. Chen, L. Liu, E. Fratini, P. Baglioni, A. Faraone, and E. Mamontov, Observation of fragile-to-strong dynamic crossover in protein hydration water, Proc. Natl. Acad. Sci. USA 103, 9012 (2006)

14. F. Mallamace, S.-H. Chen, M. Broccio, C. Corsaro, V. Crupi, et al., Role of the solvent in the dynamical transitions of proteins: The case of the lysozyme-water system, J. Chem. Phys. 127, 045104 (2007)

15. F. Mallamace, C. Branca, C. Corsaro, N. Leone, J. Spooren, et al., Dynamical crossover and breakdown of the StokesEinstein relation in confined water and in Methanol-Diluted bulk water, J. Phys. Chem. B 114(5), 1870 (2010) 
16. Y. Zhang, M. Lagi, D. Liu, F. Mallamace, E. Fratini, et al., Observation of high-temperature dynamic crossover in protein hydration water and its relation to reversible denaturation of lysozyme, J. Chem. Phys. 130, 135101 (2009)

17. M. Lagi, X. Chu, C. Kim, F. Mallamace, P. Baglioni, et al., The low-temperature dynamic crossover phenomenon in protein hydration water: Simulations vs. experiments, J. Phys. Chem. B 112(6), 1571 (2008)

18. P. Kumar, Z. Yan, L. Xu, M. G. Mazza, S. V. Buldyrev, et al., Glass transition in biomolecules and the liquid-liquid critical point of water, Phys. Rev. Lett. 97, 177802 (2006)

19. S.-H. Chen, Y. Zhang, M. Lagi, S.-H. Chong, P. Baglioni, and F. Mallamace, Evidence of dynamic crossover phenomena in water and other glass-forming liquids: Experiments, MD simulations and theory, J. Phys.: Condens. Matter 21, 504102 (2009)

20. F. Mallamace, M. Broccio, C. Corsaro, A. Faraone, L. Liu, C.-Y. Mou, and S.-H. Chen, Dynamical properties of confined supercooled water: an NMR study, J. Phys.: Condens. Matter 18, S2285 (2006)

21. W. Doster, S. Cusak, and W. Petry, Dynamical transition of myoglobin revealed by inelastic neutron scattering, Nature 337, 754 (1989)

22. W. Doster, The dynamical transition of proteins, concepts and misconceptions, Eur. Biophys. J. 37, 591 (2008)

23. W. Doster, S. Busch, A. M. Gaspar, M.-S. Appavou, J. Wuttke, and H. Scheer, Dynamical transition of proteinhydration water, Phys. Rev. Lett. 104, 098101 (2010)

24. S. Khodadadi, S. Pawlus, and A. P. Sokolov, Influence of hydration on protein dynamics: Combining dielectric and neutron scattering spectroscopy data, J. Phys. Chem. B 112, 14273 (2008)

25. S. Khodadadi, S. Pawlus, J. H. Roh, V. Garcia-Sakai, E. Mamontov, and A. P. Sokolov, The origin of the dynamic transition in proteins, J. Chem. Phys. 128, 195106 (2008)

26. G. Schirò, F. Natali, and A. Cupane, Physical origin of anharmonic dynamics in proteins: New insights from resolution-dependent neutron scattering on homomeric polypeptides, Phys. Rev. Lett. 109, 128102 (2012)

27. F. Mallamace, P. Baglioni, C. Corsaro, S.-H. Chen, D. Mallamace, C. Vasi, and H. E. Stanley, The influence of water on protein properties, J. Chem. Phys. 141, 165104 (2014)

28. F. Mallamace, C. Corsaro, D. Mallamace, S. Vasi, C. Vasi, H. E. Stanley, and S.-H. Chen, Some thermodynamical aspects of protein hydration water, J. Chem. Phys. 142, 215103 (2015)

29. F. Mallamace, C. Corsaro, D. Mallamace, S. Vasi, C. Vasi, and H. E. Stanley, Thermodynamic properties of bulk and confined water, J. Chem. Phys. 141, 18C504 (2014)

30. F. Mallamace, C. Corsaro, D. Mallamace, S. Vasi, C. Vasi, and G. Dugo, The role of water in protein's behavior: The two dynamical crossovers studied by NMR and FTIR techniques, Computational and Structural Biotechnology Journal 13, 33 (2015)

31. F. Mallamace, C. Corsaro, P. Baglioni, E. Fratini, and S.-H. Chen, The dynamical crossover phenomenon in bulk water, confined water and protein hydration water, J. Phys.: Condens. Matter 24, 064103 (2012)

32. F. Mallamace, S.-H. Chen, Y. Liu, L. Lobry, and N. Micali, Percolation and viscoelasticity of triblock copolymer micellar solutions, Physica A: Statistical Mechanics and its Applications 266, 123 (1999)

33. D. Russo, G. Hura, and T. Head-Gordon, Hydration dynamics near a model protein surface, Biophys. J. 86, 1852 (2004)

34. A. Ben-Naim, The role of hydrogen bonds in protein folding and protein association, J. Phys. Chem. 95, 1437 (1991)

35. V. Kocherbitov, J. Latynis, A. Misiūnas, J. Barauskas, and G. Niaura, Hydration of lysozyme studied by raman rpectroscopy, J. Phys. Chem. B 117, 4981 (2013)

36. G. Zaccai, How soft is a protein? A protein dynamics force constant measured by neutron scattering, Science 288, 1604 (2000)

37. P. W. Fenimore, H. Frauenfelder, B. H. McMahon, and F. G. Parak, Slaving: solvent fluctuations dominate protein dynamics and functions, Proc. Natl. Acad. Sci. USA 99, 16047 (2002)

38. H. Frauenfelder, P. W. Fenimore, and R. D. Young, Protein dynamics and function: Insights from the energy landscape and solvent slaving, IUBMB Life 59, 506 (2007)

39. F. Mallamace, C. Corsaro, and H. E. Stanley, A singular thermodynamically consistent temperature at the origin of the anomalous behavior of liquid water, Sci. Rep. 2, 993 (2012)

40. F. Chiti and C. M. Dobson, Amyloid formation by globular proteins under native conditions, Nat. Chem. Biol. 5, 15 (2009)

41. D. J. Selkoe, Folding proteins in fatal ways, Nature 426, 900 (2003)

42. G. Salvetti, E. Tombari, L. Mikheeva, and G. P. Johari, The endothermic effects during denaturation of lysozyme by temperature modulated calorimetry and an intermediate reaction equilibrium, J. Phys. Chem. B 106, 6081 (2002)

43. F. Mallamace, C. Corsaro, D. Mallamace, P. Baglioni, H. E. Stanley, and S.-H. Chen, A possible role of water in the protein folding process, J. Phys. Chem. B 115, 14280 (2011)

44. D. Mallamace, C. Corsaro, C. Vasi, S. Vasi, G. Dugo, and F. Mallamace, The protein irreversible denaturation studied by means of the bending vibrational mode, Physica A: Statistical Mechanics and its Applications 412, 39 (2014)

45. F. Sterpone, G. Stirnemann, and D. Laage, Magnitude and molecular origin of water slowdown next to a protein, J. Am. Chem. Soc. 134, 4116 (2012)

46. C. Mattea, J. Qvist, and B. Halle, Dynamics at the proteinwater interface from ${ }^{17} \mathrm{O}$ spin relaxation in deeply supercooled solutions, Biophys. J. 95, 2951 (2008)

47. E. Dubouè-Dijon, A. C. Fogarty, and D. Laage, Temperature dependence of hydrophobic hydration dynamics: From retardation to acceleration, J. Phys. Chem. B 118, 1574 (2014)

48. S. Pronk, E. Lindahl, and P. M. Kasson, Dynamic heterogeneity controls diffusion and viscosity near biological interfaces, Nature Communications 5, 3034 (2014) 
49. A. C. Fogarty and Damien Laage, Water dynamics in protein hydration shells: The molecular origins of the dynamical perturbation, J. Phys. Chem. B 118, 7715 (2014)

50. W. S. Price, Pulsed-field gradient nuclear magnetic resonance as a tool for studying translational diffusion (Part II): Experimental aspects, Concepts Magn. Reson. 10, 197 (1998)

51. A. Abragam, The Principles of Nuclear Magnetism, Oxford, UK: Oxford, 1961

52. S. Perticaroli, L. Comez, P. Sassi, M. Paolantoni, S. Corezzi, S. Caponi, A. Morresi, and D. Fioretto, Hydration and aggregation of lysozyme by extended frequency range depolarized light scattering, Journal of Non-Crystalline Solids 407, $472(2015)$

53. B. Jana, S. Pal, and B. Bagchi, Hydration dynamics of protein molecules in aqueous solution: Unity among diversity, J. Chem. Sci. 124 (1), 317 (2012)

54. A. S. Parmar and M. Muschol, Hydration and hydrodynamic interactions of lysozyme: effects of chaotropic versus kosmotropic ions, Biophysical Journal 97, 590 (2009)

55. A. Bizzarri, S. Cannistraro, Molecular dynamics of water at the protein-solvent interface, J. Phys. Chem. B 106, 6617 (2002)

56. W. S. Price, H. Ide, and Y. Arata, Self-diffusion of supercooled water to $238 \mathrm{~K}$ using PGSE NMR diffusion measure- ments, J. Phys. Chem. A 103, 448 (1999)

57. J. H. Simpson and H. Y. Carr, Diffusion and Nuclear Spin Relaxation in Water, Phys. Rev. 111, 1201 (1958)

58. C. Corsaro and D. Mallamace, A nuclear magnetic resonance study of the reversible denaturation of hydrated lysozyme, Physica A: Statistical Mechanics and its Applications 390, 2904 (2011)

59. D. W. G. Smith and J. G. Powles, Proton spin-lattice relaxation in liquid water and liquid ammonia, Mol. Phys. 10, 451 (1966)

60. T. DeFries and J. Jonas, Pressure dependence of NMR proton spin-lattice relaxation times and shear viscosity in liquid water in the temperature range $-15-10{ }^{\circ} \mathrm{C}, \mathrm{J}$. Chem. Phys. 66, 896 (1977)

61. E. Lang and H.-D. Lüdemann, Pressure and temperature dependence of the longitudinal proton relaxation times in supercooled water to $-87^{\circ} \mathrm{C}$ and 2500 bar, J. Chem. Phys. 67, 718 (1977)

62. N. Bloembergen, E. M. Purcell, and R. V. Pound, Relaxation effects in nuclear magnetic resonance absorption, Phys. Rev. 73, 679 (1948)

63. B. Halle and M. Davidovic, Biomolecular hydration: From water dynamics to hydrodynamics, Proc. Natl. Acad. Sci. USA 100, 12135 (2003)

64. http://webbook.nist.gov/chemistry/fluid/ 